\title{
Effects of washing pre-treatment on mercury concentration in fish tissue.
}

\begin{abstract}
The objective of this study was to examine the effect of washing pre-treatment on mercury concentration in fish fillet. Response surface methodology was used to investigate the influence of three variables, $\mathrm{pH}(1-6.5), \mathrm{NaCl}(0-1 \% \mathrm{w} / \mathrm{v})$ and exposure time $(5-30 \mathrm{~min})$ by using a three-factor central composite design. The aim was to obtain the best possible combination of these variables in order to reduce mercury in fish fillet. The experimental data were adequately fitted into a second-order polynomial model with multiple regression coefficients $(\mathrm{R}(2))$ of 0.961 . The results indicated that the reduction of mercury in fish flesh significantly depends on the $\mathrm{pH}$ of the solution used. The overall optimal condition resulting in the maximum mercury reduction in fish fillet was obtained at a combined level $\mathrm{pH}$ of 2.79, $\mathrm{NaCl}$ of $0.5 \%$ and exposure time of $13.5 \mathrm{~min}$. The optimized protocol produced a solution that can reduce mercury from raw fish fillet up to $81 \%$.
\end{abstract}

Keyword: Atomic absorption spectrometry (AAS); Heavy metals-mercury; Fish. 